\title{
Phylogenetic Analysis of Dioecy in Monocotyledons
}

\author{
George D. Weiblen, Ryan K. Oyama, and Michael J. Donoghue
}

Department of Organismic and Evolutionary Biology, Harvard University Herbaria, 22 Divinity Avenue, Cambridge, Massachusetts 02138

Submitted February 17, 1999; Accepted August 13, 1999

A BST RACT: Surveys of plant breeding systems in angiosperm families have shown a significant association between monoecy and dioecy, and researchers have proposed that dioecy has tended to evolve from monoecy. We evaluated this hypothesis in the context of a phylogeny of 918 monocotyledons assembled from 19 published trees. Binary and multistate breeding system characters were mapped onto a set of composite trees, and alternative models of character change were compared using maximum likelihood. Over a range of tree topologies and optimizations, we found three to eight times as many changes from hermaphroditism to dioecy as we did from monoecy to dioecy. Also, the rate at which monoecy gave rise to dioecy was not significantly higher than the rate at which hermaphroditism gave rise to dioecy. Our analysis implies that the correlation of monoecy and dioecy in angiosperm families does not reflect a preponderance of changes from monoecy to dioecy. Instead, we postulate that the family-level correlation results from the clustering of breeding system changes in the underlying phylogeny. Our results suggest renewed attention to modeling the transition from hermaphroditism to dioecy, possibly involving transient intermediates such as gynodioecy.

Keywords: breeding systems, dioecy, monocotyledons, phylogeny, parsimony, maximum likelihood.

Beginning with Darwin (1877), evolutionary biologists have tried to explain the origin and significance of dioecy in flowering plants. Although the majority of angiosperm species are hermaphroditic, approximately $6 \%$ are dioecious, with sexes segregated in separate individuals (Renner and Ricklefs 1995). Whether the evolution of dioecy results from selection for outcrossing, as Darwin suggested, or from sexual selection on separate components of male and female fitness has been a subject of considerable debate

\footnotetext{
* To whom all correspondence should be addressed. Present address: Department of Zoology, Michigan State University, East Lansing, Michigan 48824; e-mail: gweiblen@pilot.msu.edu.
}

Am. Nat. 2000. Vol. 155, pp. 46-58. (C) 2000 by The University of Chicago. 0003-0147/2000/15501-0005\$03.00. All rights reserved. (articles in the American Naturalist include Thomson and Barrett 1981; Bawa 1982; Givnish 1982; Lloyd 1982; Ross 1982; Willson 1982, 1991; Baker 1984).

In the literature on the evolution of dioecy, two general approaches are apparent. The first concentrates on modeling selection at the population level (Charnov 1982). Charlesworth and Charlesworth (1978), for example, modeled a pathway to dioecy involving the invasion of a hermaphroditic population by male-sterile individuals (gynodioecy) followed by selection against female function in the remaining hermaphrodites (also see Ross 1982; Schultz 1994). The second approach aims to identify patterns in the distribution of breeding systems across angiosperms or seed plants in general. For the most part, such studies have focused on correlations between the taxonomic distribution of dioecy and other attributes. Renner and Ricklefs (1995, p. 604), for example, in a study focused on genera within angiosperm families, found that "the single most important predictor of a group's tendency to acquire dioecy is the presence of monoecy in the group." On the basis of this association they argued that "dioecy appears to have evolved most frequently via monoecy, perhaps through divergent adjustments of floral sex ratios between individual plants" (Renner and Ricklefs 1995, p. 596; also see Lewis 1942; Westergaard 1958). Regardless of approach, the pathways described envision different kinds and numbers of evolutionary steps separating alternative breeding systems (fig. 1).

In broad comparative studies, correlations have also been documented between dioecy and self-incompatibility, heterostyly, insect pollination, bird dispersal, growth form, and living on islands (e.g., Bawa 1980, 1982; Givnish 1982; Renner and Ricklefs 1995; Sakai et al. 1995). However, the interpretation of such correlations is complicated. As Baker (1984) noted, the presence of two traits within a taxonomic group (e.g., heterostyly and dioecy) does not necessarily mean that one arose from the other. And, as Donoghue (1989) illustrated in testing a hypothesis advanced by Givnish (1980), counting the number of taxa (species, genera) with a particular combination of traits (e.g., fleshy propagules and dioecy) can overestimate the number of times that a particular sequence of evolutionary change (first fleshy propagules, then dioecy) probably oc- 


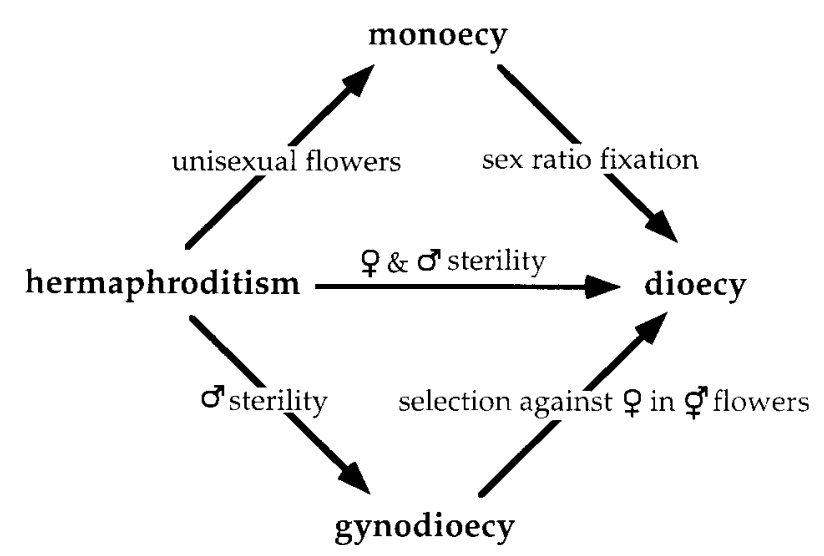

Figure 1: Some evolutionary pathways to dioecy in flowering plants. Renner and Ricklefs (1995) proposed that dioecy evolves from monoecy through the genetic fixation of sex ratios between individuals. Charlesworth and Charlesworth (1978) proposed a model that invoked the spread of male-sterile individuals in a hermaphroditic population (gynodioecy) followed by selection against femaleness in the remaining hermaphrodites. Dioecy may also evolve from hermaphroditism through the expression of male and female sterility genes in separate individuals (Ross 1982).

curred. Thomson and Brunet (1990) also emphasized the use of phylogenetic trees in studying plant breeding systems, and numerous examples have appeared (reviewed by Weller and Sakai 1999).

Renner and Ricklefs (1995, p. 604) clearly appreciated these points, noting that although "the general direction of evolutionary change is likely to be from monoecy to dioecy ... phylogenetic analyses of individual examples are needed to support this assumption." Here we report the results of a phylogenetic analysis of breeding system evolution, focusing specifically on pathways to dioecy in a composite phylogeny of monocotyledons. We focus on monocotyledons because this is a large clade (over onequarter of all angiosperm species) that has recently received considerable phylogenetic study (e.g., see Rudall et al. 1995). In particular, we address the following questions: How many times have monoecy, dioecy, and other breeding systems evolved in monocots? Have shifts among some breeding systems occurred more often than others? And has dioecy evolved most often from hermaphroditism and gynodioecy, as modeled by Charlesworth and Charlesworth (1978) and others, or from monoecy, as inferred by Renner and Ricklefs (1995) based on taxonomic correlations? More generally, we consider the conceptual link between phylogenetic analysis of breeding system evolution and models of evolution at the population level.

\section{Methods}

Tree Assembly

We assembled a large phylogenetic tree of monocotyledons by combining a number of published trees into one. The aim of this exercise was to increase the number of taxa in the tree so as to enhance the power of phylogenetic analyses to detect and explain general evolutionary patterns. Composite trees (e.g., Sillen-Tulberg 1988, 1993; Donoghue 1989; Janz and Nylin 1998; Rolland et al. 1998) provide a broader look at character evolution than the individual studies on which they are based. However, as we argue in the discussion, they compound uncertainty and may increase bias because of uneven taxonomic sampling of the underlying phylogeny (also see Sanderson et al. 1998). For this reason, analyses aimed at exploring the sensitivity of results to tree topology (Losos 1995; Weller et al. 1995; Donoghue and Ackerly 1996; Ackerly and Donoghue 1998; Donoghue et al. 1998) and taxon sampling (Ree and Donoghue 1998) are especially critical in such cases. However, comparative analyses conducted on very large trees may also be particularly robust to changes in underlying topology (Donoghue and Ackerly 1996).

In assembling a monocot phylogeny for our analyses, we relied on published tree topologies without evaluating support for particular clades or attributes of the underlying data sets. When more than one tree had been published for a particular group, we used several criteria in choosing among them.

First, only trees based on analyses of character matrices under some optimality criterion were considered; otherwise, no method of phylogenetic inference was preferred, although most published studies are based on maximum parsimony. Second, no one type of data was preferred; instead, we selected studies including either the highest number of informative characters or those based on combined analysis of data from different sources (de Queiroz et al. 1995). For example, we selected a starting monocot tree based on combined molecular and morphological data (table 1), as opposed to a tree based on morphological characters alone (Stevenson and Loconte 1995). Third, we chose trees containing maximum information about phylogenetic relationships, namely, those with the most taxa and greatest resolution. In general, this meant choosing a published most parsimonious (MP) tree, as opposed to a consensus tree. Fourth, we did not include phylogenies in which there were no deviations from hermaphroditism (e.g., Haemodoraceae: Simpson 1990; Pontederiaceae: Kohn et al. 1996; Triticeae: Mason-Gamer and Kellogg 1996). Finally, monophyletic groups were pruned from less inclusive phylogenies and grafted into more inclusive phylogenies in cases where the placement of groups was unambiguous and where grafting increased the number of 
Table 1: Phylogenies used to construct a composite tree of monocots.

\begin{tabular}{|c|c|c|}
\hline Clade & Reference & $\begin{array}{l}\text { Data matrix } \\
\text { (tree description) }\end{array}$ \\
\hline 1. Monocotyledons & Chase et al. $1995 b$, figure 4 & $r b c \mathrm{~L} \&$ morphology ( 1 of $96 \mathrm{MP}$ trees) \\
\hline 2. Asparagales & Chase et al. $1995 a$, figure 2 & $r b c \mathrm{~L}(1$ of $11468 \mathrm{MP}$ trees $)$ \\
\hline 3. Liliales & Chase et al. $1995 a$, figure 3 & $r b c \mathrm{~L}$ ( 1 of $11468 \mathrm{MP}$ trees) \\
\hline 4. Stemonoids & Chase et al. $1995 a$, figure 1 & $r b c \mathrm{~L}$ ( 1 of $11468 \mathrm{MP}$ trees $)$ \\
\hline 5. Dasypogonaceae & Chase et al. $1995 a$, figure 4 & $r b c \mathrm{~L}$ ( 1 of $11468 \mathrm{MP}$ trees $)$ \\
\hline 6. Commelinids & Linder and Kellogg 1995, figure 3 & $r b c \mathrm{~L} \&$ morphology ( 1 of $96 \mathrm{MP}$ trees) \\
\hline 7. Arecaceae & Uhl et al. 1995 , figures 2,3 & cpDNA RFLPs \& morphology ( 1 of 98 MP trees) \\
\hline 8. Ariflorae & French et al. 1995, figure 1 & cpDNA RFLPs (strict consensus of 1344 trees) \\
\hline 9. Alismatideae & Les et al. 1997, figure 2 & $r b c \mathrm{~L}$ (strict consensus of $32 \mathrm{MP}$ trees) \\
\hline 10. Agavaceae & Bogler and Simpson 1996, figure 3 & ITS rDNA (strict consensus of $4 \mathrm{MP}$ trees) \\
\hline 11. Pandanaceae & Cox et al. 1995, figure 6 & Morphology (MP tree) \\
\hline 12. Zingiberales & Kress 1995, figure 6 & $r b c \mathrm{~L} \&$ morphology ( 1 of $2 \mathrm{MP}$ trees) \\
\hline 13. Poales & Kellogg and Linder 1995, figure 12 & $\begin{array}{l}r b c \mathrm{~L}, r p o \mathrm{C} 2, \mathrm{cpDNA} \text { RFLPs \& morphology (strict } \\
\text { consensus of } 99 \mathrm{MP} \text { trees) }\end{array}$ \\
\hline 14. Cyperales & Simpson 1995, figure 1 & Morphology (MP tree) \\
\hline 15. Graminae & Kellogg and Campbell 1987, figure 28.2 & Morphology ( 1 of 7 MP trees) \\
\hline 16. Pooideae & Kellogg and Watson 1993, figure 10 & Morphology ( 1 of 500 MP trees) \\
\hline 17. Bambusoideae & Kellogg and Watson 1993, figure 3 & Morphology ( 1 of 32 MP trees) \\
\hline 18. Chloridoideae & Van den Borre 1994, figure 6 & Morphology (distance tree) \\
\hline 19. Panicoideae & Kellogg and Watson 1993, figure 7 & Morphology ( 1 of 5000 MP trees) \\
\hline
\end{tabular}

Note: Figure 2 illustrates the relationships between clades in the composite tree, which is available from G. Weiblen. Trees from individual studies are deposited in TreeBASE (http://phylogeny.harvard.edu/treebase).

taxa in the composite tree. In most cases, pruning involved only the removal of outgroups, while grafting required the replacement of some taxa or small clades with clades from other studies. In addition, the following minor modifications were made: Schizachne and Sclerodactylon (Poaceae) each appeared twice in the initial composite tree because of conflicting placements in Kellogg and Watson (1993) and Van den Borre (1994). These were assigned a single position based on Watson and Dallawitz (1992). Astelia, which was omitted in pruning clade 1, was grafted into Asparagales (Chase et al. 1995a) as sister to Blandfordia, based on its position in Chase et al. (1995b).

Figure 2 shows an overview of the composite tree used as a starting point in our analyses. A Nexus file containing the composite tree is available from the first author; this file can be downloaded to MacClade (Maddison and Maddison 1992) to view the trees and character scorings used in this study. The individual trees underlying our composite tree can be obtained from TreeBASE at http:// phylogeny.harvard.edu/treebase. The phylogenetic studies represented in the composite tree include 91 of the 138 instances of dioecy $(66 \%)$ in monocot genera reported by Renner and Ricklefs (1995).

\section{Characters, States, and Scoring Issues}

To assess the frequency of transitions between monomorphic and polymorphic (mostly dimorphic) breeding sys- tems, we scored all taxa for the following binary character: (0) flowers hermaphroditic and alike in sexuality throughout the species, or (1) flowers not all alike in sexuality. The absence of functional stamens or carpels in some flowers implies the differentiation of two (or more) sexual arrangements per species. Polymorphism involves unisexual flowers of two forms or, in some cases, a combination of unisexual and hermaphroditic flowers arranged on the same plant or separate plants.

To investigate transitions between all possible arrangements of stamens and carpels, we scored all taxa for a character with the following nine states: (0) hermaphroditic, with flowers all alike in sexuality throughout the species; (1) monoecious, with staminate and carpellate flowers on the same plant; (2) dioecious, with staminate and carpellate flowers on different plants; (3) andromonoecious, with staminate and hermaphroditic flowers on the same plant; (4) gynomonoecious, with carpellate and hermaphroditic flowers on the same plant; (5) androdioecious, with staminate and hermaphroditic flowers on separate plants; (6) gynodioecious, with carpellate and hermaphroditic flowers on separate plants; (7) polygamomonoecious, with carpellate, staminate, and hermaphroditic flowers on the same plant; and (8) polygamodioecious, with carpellate, staminate, and hermaphroditic flowers on separate plants.

Scoring of these characters was based on published accounts, including original taxonomic descriptions, floras, 


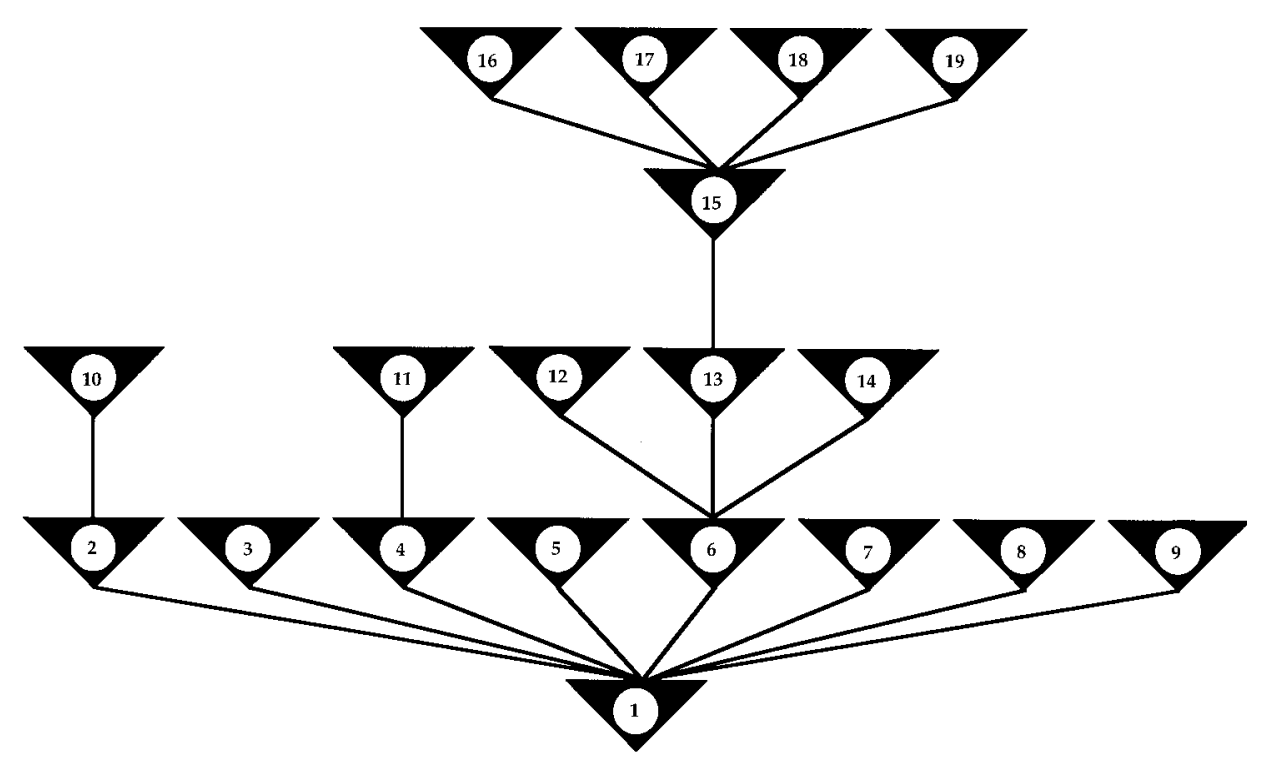

Figure 2: Summary of the connection between individual trees in the composite monocotyledon tree used in this study. This representation is meant only to indicate how individual trees are nested within each other and nothing about taxonomic ranks. Numbers refer to individual studies in table 1; see text for details on tree assembly. The composite tree can be obtained from the first author. Trees from individual studies are available at http://phylogeny.harvard.edu/treebase.

and surveys of sex expression (e.g., Yampolsky and Yampolsky 1922; Renner and Ricklefs 1995). When (rarely) conflicting accounts of breeding system were encountered, more recent citations were preferred over older citations, except when the more recent information lacked sufficient detail. We also accounted for changes in the circumscription of families and genera in an attempt to accurately connect the older literature to currently accepted names. A complete list of sources for breeding system information is available from G. Weiblen on request.

Only 17 of the terminal taxa in the composite tree were polymorphic for breeding system. However, the occurrence of more than one state in a terminal taxon is problematical for inferring ancestral states (Maddison and Maddison 1992), and we therefore split polymorphic taxa into separate clades representing the different states (Nixon and Davis 1991; see Donoghue 1994 for commentary on this procedure in phylogenetic inference). For example, Scleropogon (Poaceae) includes both monoecious and dioecious species and was represented by a single polymorphic taxon in the Chloridoideae clade (Van den Borre 1994). We split Scleropogon into two taxa, one representing the monoecious species and the other the dioecious species. This established a minimum estimate of the number of evolutionary events; if we knew more about the phylogeny of Scleropogon, we might have discovered several independent derivations of monoecy or dioecy within the group. Such splits into two taxa were carried out for Apon- ogeton, Asparagus, Blyxa, Buchloe, Centrolepidaceae, Chioncloa, Distichilis, Jouvea, Leucopoa, Soderstromia, and Zigadenus. To reflect the occurrence of three breeding systems, Bouteloua, Carex, Cortaderia, Dioscoreaceae, and Restoniaceae were each split into three taxa. Elimination of polymorphisms by this procedure expanded our composite tree from 894 to 918 taxa.

We used MacClade (Maddison and Maddison 1992) to reconstruct ancestral breeding systems and infer state changes on a completely resolved composite tree under a range of assumptions, as well as to conduct the sensitivity analyses described below. To avoid difficulties in inferring ancestral states with polytomies (Maddison 1989), we used the option in MacClade to randomly resolve polytomies, which resulted in completely dichotomous trees. This procedure should not systematically bias inferred patterns of character evolution, but we nevertheless conducted sensitivity analyses using randomly generated trees for the same set of 918 taxa. We compared changes in 100 dichotomous resolutions of our starting composite tree. We also compared these results with changes inferred on 100 randomly generated trees in order to explore the sensitivity of our results to major rearrangements in tree topology (cf. Donoghue and Ackerly 1996). Measures of homoplasy on these trees were also compared with averages obtained from permuted data (cf. Sanderson 1991). Permutation involved the random and repeated assignment of the observed character state frequencies to the terminal taxa. It 
was not feasible to compute all possible reconstructions of ancestral states using the equivocal cycling option in MacClade, owing to the size of the tree and the existence of multiple equally parsimonious solutions at some nodes. Instead, the frequencies of unambiguous character changes under ACCTRAN and DELTRAN optimizations were calculated for each dichotomous tree.

Alternative models of character change were also compared using a maximum likelihood method for discrete characters that does not require the reconstruction of ancestral states (Pagel 1994, 1997). We used two-, three-, and four-state characters with versions $\mathrm{d} 3$ and $\mathrm{d} 4$ of the program Discrete (Pagel 1994, 1997) to test particular hypotheses regarding the direction of breeding system evolution. In the case of the binary character, a log-likelihood ratio test compared models of evolution assuming equal versus unequal transition probabilities among monomorphic and polymorphic breeding systems. Three- and fourstate characters were used to test the expectation derived from Renner and Ricklefs (1995) that transitions from hermaphroditism to dioecy may be less frequent than transitions from monoecy to dioecy. A four-state character was defined: (0) hermaphroditism, (1) monoecy, (2) dioecy, or (3) any other breeding system. We compared the likelihood of a model assuming unrestricted parameters with a model assuming $q 02=q 12$, where $q a b$ is the probability of change from state $a$ to state $b$ along a branch of the composite tree. In the case of nested models, the likelihood ratio statistic is $\chi^{2}$ distributed with the degrees of freedom equal to the difference in the number of parameters in the models under comparison. A character with states (0) hermaphroditism, (1) dioecy, or (2) any other breeding system made possible a simplified test of the likelihood of transitions to dioecy from hermaphroditism or from any other breeding system. In this case, we compared the likelihoods of a model assuming no restrictions with a model with $q 01=q 21$. In all likelihood analyses, branch lengths were assumed to be equal. At least in the case of large trees, branch length modifications are not likely to have a significant impact on the estimated likelihoods (Ree and Donoghue 1999). Changing the number and/or length of branches with a particular state (e.g., to approximate underlying percentages of species with different conditions) can have an important impact on likelihood estimates (Ree and Donoghue 1999). We did not explore such modifications, however, because the percentage of dioecious taxa in our composite tree (ca. 10\%; table 2 ) is reasonably close to estimates for angiosperms as a whole $(7 \%-10 \%$ for genera and $6 \%$ for species; Yampolsky and Yampolsky 1922; Renner and Ricklefs 1995).

Assumptions about character weighting were evaluated under parsimony using step matrices (Maddison and Maddison 1992; Maddison 1994) and "cost-change" analysis
Table 2: Number and frequency of taxa with different breeding systems in a binary and a multistate character on a composite tree of monocotyledons.

\begin{tabular}{cllc}
\hline State & \multicolumn{1}{c}{ Breeding system } & $\begin{array}{c}\text { Number } \\
\text { of taxa }\end{array}$ & Frequency \\
\hline Two states: & & & \\
0 & Monomorphic & 614 & .669 \\
1 & Dimorphic/polymorphic & 304 & .331 \\
Nine states: & & & \\
0 & Hermaphroditic & 614 & .669 \\
1 & Monoecious & 132 & .144 \\
2 & Dioecious & 91 & .099 \\
3 & Andromonoecious & 59 & .064 \\
4 & Gynomonoecious & 5 & .005 \\
5 & Androdioecious & 1 & .001 \\
6 & Gynodioecious & 5 & .005 \\
7 & Polygamomonoecious & 7 & .008 \\
8 & Polygamodioecious & 4 & .004 \\
\hline
\end{tabular}

(Ree and Donoghue 1998). We explored how great a cost must be imposed on changes from hermaphroditism to dioecy for the frequency of inferred monoecy-to-dioecy changes to exceed hermaphroditism-to-dioecy changes. For the sake of simplicity, we used a character with three states: (0) hermaphroditism, (1) dioecy, and (2) monoecy plus all other breeding systems. We then defined weights $x, y$, and $z$ as the costs of 02,21 , and 01 changes, respectively. Next, we increased $z$ relative to $x$ and $y$ by increments, keeping the cost of $x$ and $y$ equal and ensuring that each step matrix satisfied the triangle inequality. The cost of reversals was assumed to be equal to one in all cases.

\section{Results}

Under parsimony we inferred that the ancestor of monocots was hermaphroditic. In our focal monocot tree, we recorded 66 unambiguous changes from monomorphic to polymorphic breeding systems and 24 reversals in an ACCTRAN optimization. The DELTRAN optimization yielded 71 changes from monomorphism to polymorphism and 19 reversals. On 100 dichotomous resolutions of the starting composite tree, we found an average of 59 unambiguous gains of polymorphism (min-max: 54-62) and 18 losses (min-max: 17-19). In contrast, we obtained an average of 162 gains (min-max: 127-200) and 12 losses (min-max: 4-28) on 100 random trees.

Shifts between the nine breeding systems on our focal composite tree are summarized in table 3 . We found about eight times as many changes to dioecy from hermaphroditism than from monoecy (26-35 vs. 3-4 times). There were 9-11 reversals from dioecy to monoecy; more than expected by chance and three times the number of shifts from monoecy to dioecy. The six reversals from dioecy to 
hermaphroditism were more common than expected by chance and also more common than reversals from monoecy, despite the presence of 50\% more monoecious than dioecious taxa in the composite tree (table 2). To gauge relative frequencies, we divided the number of gains of dioecy from either hermaphroditism or monoecy by the number of terminal taxa bearing each state. By this measure, the frequency of changes to dioecy in hermaphroditic clades $(0.03)$ was three times greater than in monoecious clades (0.01). Gynodioecy was seen to have evolved from hermaphroditism at least three times independently but did not appear to have given rise to dioecy. The evolution of andromonoecy from hermaphroditism occurred repeatedly (16-18 times) but was limited to the grass clade (Poaceae). Shifts from andromonoecy to hermaphroditism, monoecy, and gynomonoecy within the grasses also occurred multiple times (table 3 ).

Consistency index (CI) was very low for binary and multistate characters, indicating that particular breeding systems evolved repeatedly in monocots. The CI of 0.01 for the binary character on our focal tree was still far greater than under a permuted distribution of states (mean $\mathrm{CI}=0.004$ ), indicating significant autocorrelation of breeding systems on the composite tree. Likewise, the ninestate character showed less homoplasy $(\mathrm{CI}=0.06)$ than when the distribution of states was permuted $(\mathrm{CI}=0.03)$.

Comparison of dichotomous composite trees with randomly generated trees (table 4) showed that the results are influenced by tree topology. As expected, breeding systems showed greater autocorrelation in multiple alternative resolutions of the composite tree than in random trees. More precisely, monoecy and dioecy originated less often and characterized larger clades in composite trees than in random trees. However, reversals from dioecy and andromonoecy to hermaphroditism and monoecy, respectively, were more common in composite trees than in random trees.

With Discrete we found no significant difference in the probability of changes between monomorphic (0) and polymorphic (1) breeding systems (likelihood ratio, $\mathrm{LR}=0.0007, \mathrm{df}=1)$. In other words, the estimated rates of change $(q 01=0.0530, q 10=0.0605)$ were not significantly more likely to yield the observed data than a restricted model with equal rates of change $(q 01=q 10)$. In the case of three states, we could not reject the hypothesis that the rate of change from hermaphroditism to dioecy is equal to the rate of change from any other breeding system to dioecy ( $\mathrm{LR}=0.0327, \mathrm{df}=1)$. Nor could we reject the hypothesis that changes from hermaphroditism to dioecy $(q 02=0.0286)$ were as probable as changes from monoecy to dioecy $(q 12=0.0233)$ in the case of four states $(\mathrm{LR}=0.0008, \mathrm{df}=1)$. Rates of change among breeding systems are summarized in tables 5, 6, and 7 .
We also compared alternative step matrices for the three-state character under parsimony. We found that the frequency of changes from monoecy to dioecy only exceeds that of hermaphroditism to dioecy when we assume that hermaphroditism-to-dioecy changes were eight times more costly than monoecy-to-dioecy changes. Eliminating all of the inferred hermaphroditism-to-dioecy changes from the tree required that they be nine times more costly than monoecy-to-dioecy changes. In this case, the ancestors of most hermaphroditic clades were inferred to be monoecious. When we assumed that hermaphroditism to dioecy changes involved two steps, while monoecy to dioecy changes involved one step (as per the Renner and Ricklefs [1995] model; see fig. 1), we observed that changes to dioecy from hermaphroditism still outnumbered changes from monoecy by a ratio of $17: 10$.

\section{Discussion}

Considering the number of evolutionary changes inferred under parsimony (tables 3,4 ) and the rates of change inferred under maximum likelihood (tables 5, 6, 7), we conclude that dioecy probably evolved more often from hermaphroditism than it did from monoecy, or at least that the rate at which monoecy gave rise to dioecy is no higher than the rate at which hermaphroditism gave rise to dioecy. These findings differ from earlier conclusions based on taxonomic ranks (Lewis 1942; Westergaard 1958; Renner and Ricklefs 1995). On the basis of the correlation of monoecious and dioecious genera in flowering plant families, these authors hypothesized the common occurrence of a two-step evolutionary pathway involving shifts from hermaphroditism to monoecy followed by changes from monoecy to dioecy (fig. 1). Lewis (1942, p. 52) stated this model clearly when he wrote, "the change from hermaphroditism requires more steps than from monoecy."

One possible explanation for the difference in conclusions is that the correlation observed across all angiosperms is not present in monocots alone. In other words, if Renner and Ricklefs (1995) had focused only on monocots, they would not have reported a significant correlation and would therefore not have reached the same evolutionary conclusion. This is not the case, however. Sixteen of 60 monocot families include both monoecious and dioecious genera, and of the remaining 44 families in which monoecy and dioecy are not coincident, seven families with monoecious genera lack dioecy and seven with dioecious genera lack monoecy. As in angiosperms as a whole, the association of monoecy and dioecy within families of monocots is statistically significant (goodness of fit with Williams's correction $=15.74, P<.001)$.

The reason we have reached different conclusions is that the occurrence of monoecy and dioecy within monocot 
Table 3: Evolutionary changes among nine breeding systems in monocotyledons: unambiguous changes under ACCTRAN (and DELTRAN) optimizations on the focal composite tree

\begin{tabular}{lccccccccc}
\hline & Herm & Mono & Dio & Androm & Gynom & Androd & Gynod & Polym & Polyd \\
\hline Herm & $\ldots$ & $9(17)$ & $26(35)$ & $16(18)$ & $3(2)$ & $1(1)$ & $3(3)$ & $3(4)$ & $2(2)$ \\
Mono & $2(1)$ & $\ldots$ & $3(4)$ & $2(2)$ & $0(0)$ & $0(0)$ & $0(0)$ & $0(0)$ & $0(0)$ \\
Dio & $6(6)$ & $9(11)$ & $\ldots$ & $0(1)$ & $0(1)$ & $0(0)$ & $1(0)$ & $0(0)$ & $0(0)$ \\
Androm & $11(10)$ & $4(4)$ & $0(0)$ & $\ldots$ & $2(2)$ & $0(0)$ & $0(0)$ & $1(1)$ & $0(0)$ \\
Polyd & $0(0)$ & $1(1)$ & $0(0)$ & $0(0)$ & $0(0)$ & $0(0)$ & $0(0)$ & $0(0)$ & $\ldots$ \\
\hline
\end{tabular}

Note: Ancestral and derived states are listed in the left column and top row, respectively. Transitions from androdioecy, gynodioecy, polygamomonoecy, or polygamodioecy to other states were not detected and are omitted from the ancestral state column. Transitions from hermaphroditism to dioecy, and from monoecy to dioecy, are italicized. Herm = hermaphroditic, $\quad$ Mono $=$ monoecious, Dio $=$ dioecious, Androm $=$ Andromonoecious, $\quad$ Polyd $=$ Polygamodioecious, Gynom $=$ Gynomonoecious, Androd $=$ Androdioecious, Gynod $=$ Gynodioecious, Polym $=$ Polygamomonoecious.

families does not mean that monoecy gave rise to dioecy. Phylogenetic relationships within such families often imply multiple origins of dioecy, often from hermaphroditism. As an illustration, consider a phylogeny of the palm family (Arecaceae; fig. 3). In this case, we see two unambiguous changes from monoecy to dioecy but four shifts from hermaphroditism to dioecy or polygamodioecy. As in monocots as a whole, the more common change is not the pathway based on taxonomic ranks (Lewis 1942; Renner and Ricklefs 1995). In the taxonomic correlation, palms are simply scored as a family having both monoecy and dioecy present, and details on changes within the group are unspecified. If the rank of family is ignored, and instead we count the number of changes inferred on the phylogeny, we obtain a rather different picture. Dioecy sometimes evolves from monoecy, but it evolves more often from hermaphroditism.

This explains how a phylogenetic approach yields a different answer, but why is there a strong positive correlation at the family level in the first place? We think that the taxonomic correlation results from the clustering of changes in breeding system within particular clades of monocots. That is, breeding system seems to be especially labile within certain clades, such that shifts to different breeding systems occur in relative proximity on the tree (see Sanderson 1991). When breeding system changes are clustered in the tree, almost any taxonomic circumscription of families - whether based on numbers of taxa, breeding systems, or other attributes-would tend to group monoecious and dioecious species within families. We believe that the correlation is best interpreted not in terms of a tendency for dioecy to evolve from monoecy but, rather, as a tendency for changes in breeding system to be phylogenetically clustered, perhaps as a consequence of underlying developmental genetic changes in certain lineages.

Although the distribution of homoplasy in monocot breeding systems is intriguing, it will be difficult to explain without more comparative data on the developmental basis and genetics of sex determination. LeRoux and Kellogg (1999) addressed this question in the grass subfamily Panicoideae (clade 19 in fig. 1) and suggested a mechanism for sex determination based on maize genetics and patterns of sex expression in the tribe Andropogoneae. DeLong et al. (1993) identified in maize a single gene ( $t s 2$ ) that affects the selective abortion of gynoecia through cell death to produce female-sterile inflorescences. LeRoux and Kellogg (1999) proposed that the origin of a cell death pathway involving ts 2 could account for the shift from hermaphroditism to andromonoecy in the Panicoideae. Repeated

Table 4: Evolutionary changes among nine breeding systems in monocotyledons: average unambiguous changes under ACCTRAN for 100 alternative dichotomous composite trees (and 100 randomly generated trees)

\begin{tabular}{lccccccccc}
\hline & Herm & Mono & Dio & Androm & Gynom & Androd & Gynod & Polym & Polyd \\
\hline Herm & $\ldots$ & $7(88)$ & $22(62)$ & $15(41)$ & $2(3)$ & $1(1)$ & $4(4)$ & $3(5)$ & $2(3)$ \\
Mono & $0(1)$ & $\ldots$ & $3(1)$ & $2(1)$ & $0(1)$ & $0(0)$ & $0(0)$ & $0(0)$ & $0(0)$ \\
Dio & $3(0)$ & $9(1)$ & $\ldots$ & $0(0)$ & $0(0)$ & $0(0)$ & $0(0)$ & $0(0)$ & $0(0)$ \\
Androm & $10(0)$ & $4(0)$ & $0(0)$ & $\ldots$ & $2(0)$ & $0(0)$ & $0(0)$ & $1(0)$ & $0(0)$ \\
Polyd & $0(0)$ & $1(0)$ & $0(0)$ & $0(0)$ & $0(0)$ & $0(0)$ & $0(0)$ & $0(0)$ & $\ldots$ \\
\hline
\end{tabular}

Note: Ancestral and derived states are listed in the left column and top row, respectively. Transitions from androdioecy, gynodioecy, polygamomonoecy, or polygamodioecy to other states were not detected and are omitted from the ancestral state column. Transitions from hermaphroditism to dioecy, and from monoecy to dioecy, are italicized. Abbreviations are identified in table 3. 
Table 5: Rates of change in monocot breeding systems estimated by maximum likelihood under less (and more) restricted models of evolution using Discrete (Pagel 1994, 1997)

\begin{tabular}{lcc}
\hline & \multicolumn{2}{c}{ Rate of change } \\
\cline { 2 - 3 } State & Monomorphic (0) & Polymorphic (1) \\
\hline Two states: & & \\
$\quad$ Monomorphic & $\ldots$ & .0530 \\
& & $(.0549)$ \\
Polymorphic & .0605 & $\ldots$ \\
& $(.0549)$ & \\
\hline
\end{tabular}

Note: Assuming equal branch lengths, $q a b$ is the probability of change from state $a$ to state $b$ along a branch of the composite tree. The more restricted model was defined as $q 01=q 10$.

modification of such a pathway could result in the clustering of particular breeding system changes in the tree. In the Andropogoneae, for example, we inferred 10 reversals from andromonoecy to hermaphroditism, more than expected by chance and possibly reflecting the repeated loss of a functional cell death pathway. For most monocot clades, genetic and developmental data are insufficient to allow a general explanation for patterns in monocot breeding system evolution.

At this point it is important to reflect on sources of uncertainty and caution against the misinterpretation of our analyses. First, it is definitely true that published phylogenies are not entirely correct, and inaccuracies are compounded in the assembly of a composite tree and in the arbitrary resolution of polytomies (Donoghue and Ackerly 1996; Sanderson et al. 1998; Ree and Donoghue 1999). Also, as discussed in "Methods," we have not taken into account the strength of support for relationships or conflicts among trees derived from different datasets or analyses. Second, the sampling of taxa may be inadequate to detect particular changes or significant differences in rates of change, although a composite tree does help in this regard (Ree and Donoghue 1999). Our study was, of course, limited by the availability of phylogenetic analyses. For lack of sufficiently broad phylogenetic studies, we were unable to consider in detail several clades with considerable variation in breeding system, such as sedges (Cyperaceae) and yams (Dioscoreaceae). Third, there are a variety of problems associated with the circumscription of characters and states and the scoring of taxa, especially when sex expression is either poorly characterized or difficult to categorize (e.g., sex changes in Arisaema; Bierzychudek 1984). Finally, possible biases associated with parsimony analysis of character evolution, and the existence of multiple equally parsimonious solutions in some cases, also contribute to uncertainty (e.g., Swofford and Maddison
Table 6: Rates of change in monocot breeding systems estimated by maximum likelihood under less (and more) restricted models of evolution using Discrete (Pagel 1994, 1997)

\begin{tabular}{lccc}
\hline \multicolumn{4}{c}{ Rate of change } \\
State & Herm (0) & Dioecy (1) & Other (2) \\
\hline $\begin{array}{c}\text { Three states: } \\
\text { Herm }\end{array}$ & $\ldots$ & .0292 & .0279 \\
& & $(.0422)$ & $(.0245)$ \\
Dioecy & .0610 & & .0186 \\
& $(.0547)$ & $\ldots$ & $.0240)$ \\
Other & .0685 & .1418 & $\ldots$ \\
& $(.0847)$ & $(.0422)$ & \\
\hline
\end{tabular}

Note: Assuming equal branch lengths, $q a b$ is the probability of change from state $a$ to state $b$ along a branch of the composite tree. The more restricted model was defined as $q 02=q 12$. Transitions to dioecy are in italics. Abbreviations are identified in table 3.

1992; Collins et al. 1994; Frumhoff and Reeve 1994; Maddison 1994; Schultz et al. 1996; Ree and Donoghue 1998).

While none of these problems can be circumvented entirely, we have tried in several ways to contain them or at least to understand their effects. Regarding tree uncertainties, we explored the sensitivity of the results to alternative resolutions of polytomies in the composite tree and compared random trees and randomly shuffled characters (cf. Donoghue and Ackerly 1996). Our discussion centers on findings that are robust to the changes in tree topology that we explored. Concerning the second point, and in view of the fact that approximating the underlying distribution of character states has been identified as a critical factor (Ree and Donoghue 1999), it is noteworthy that the percentage of dioecious taxa in our composite tree (table

Table 7: Rates of change in monocot breeding systems estimated by maximum likelihood under less (and more) restricted models of evolution using Discrete (Pagel 1994, 1997)

\begin{tabular}{lcccc}
\hline & \multicolumn{4}{c}{ Rate of change } \\
\cline { 2 - 5 } State & Herm (0) & Monoecy (1) & Dioecy (2) & Other (3) \\
\hline Four states: & & & & \\
Herm & $\ldots$ & .0034 & .0286 & .0255 \\
& & $.0038)$ & $(.0266)$ & $(.0256)$ \\
Monoecy & .0000 & $\ldots$ & .0233 & .0199 \\
& $(.0000)$ & & $.0266)$ & $(.0265)$ \\
Dioecy & .0797 & .1348 & & .0067 \\
& $(.0793)$ & $(.1346)$ & $\ldots$ & $(.0030)$ \\
Other & .1983 & .0362 & .0232 & $\ldots$ \\
& $(.1985)$ & $(.0357)$ & $(.0262)$ & \\
\hline
\end{tabular}

Note: Assuming equal branch lengths, $q a b$ is the probability of change from state $a$ to state $b$ along a branch of the composite tree. The more restricted model was defined as $q 02=q 12$. Transitions to dioecy are in italics. Abbreviations are identified in table 3 . 
breeding system

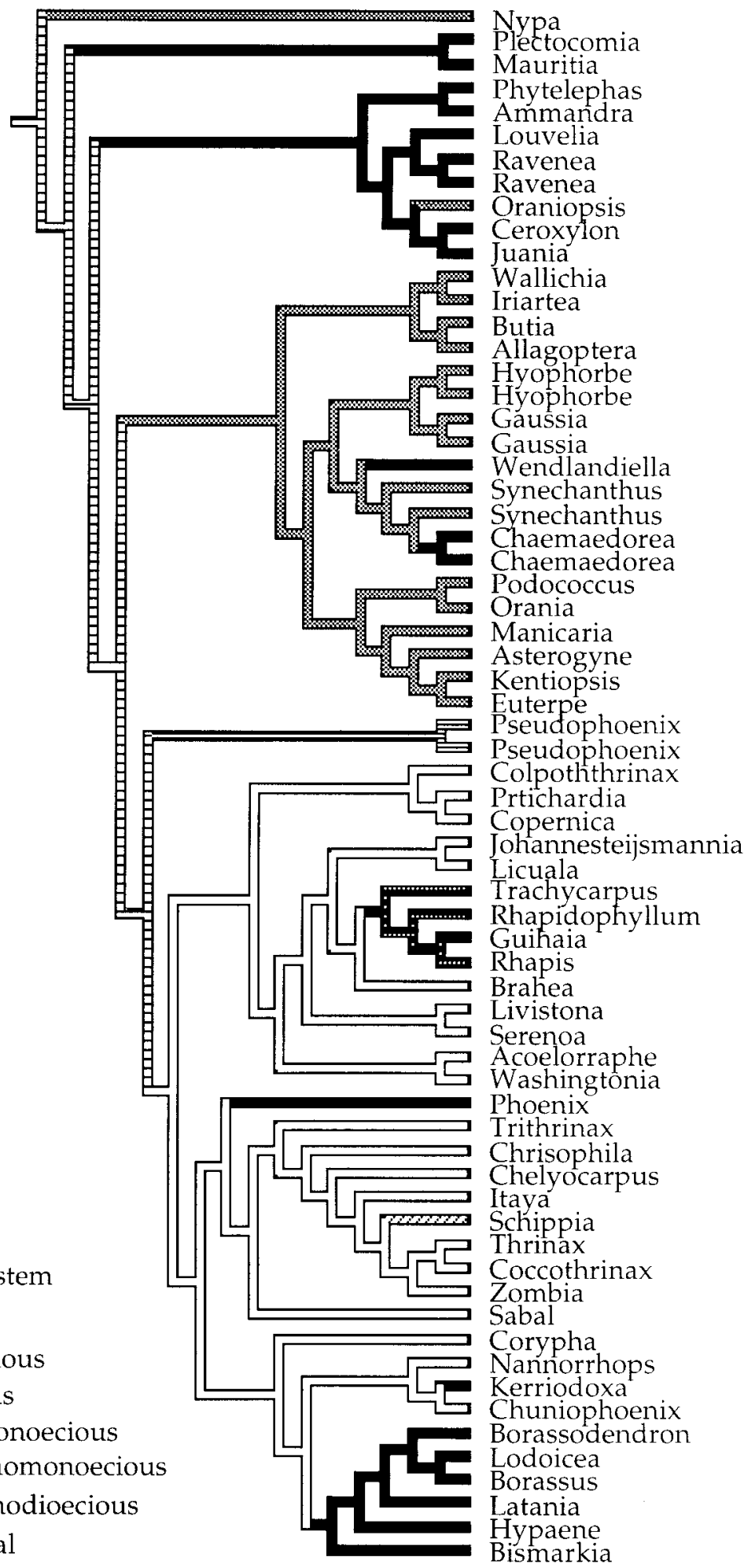

Figure 3: Breeding system evolution inferred under parsimony (using MacClade; Maddison and Maddison 1992) on a phylogeny for the palm family (Uhl et al. 1995; with random resolution of polytomies). There were two unambiguous changes from monoecy to dioecy and four unambiguous changes from hermaphroditism to dioecy or polygamodioecy. 
2) is close to estimates for angiosperms as a whole. To address character coding and scoring issues mentioned in the third point, we considered alternative character circumscriptions (binary and multistate). Finally, concerning inference issues, we focused on unambiguous changes under parsimony while also recording the range obtained using different optimization procedures. Although such sensitivity analyses could be extended in a variety of ways, the results of comparative analyses conducted on very large phylogenies, entailing many relevant state changes, are likely to be robust to considerable change in tree topology, alternative scorings, and character coding schemes (Donoghue and Ackerly 1996). We are therefore confident that the main results of this study, especially the finding that dioecy originates more often from hermaphroditism than from monoecy, will be upheld in further tests.

One major conceptual difficulty remains. We observed many direct transitions from hermaphroditism to dioecy, but is it possible that we missed intermediate stages? Perhaps we failed to include extant or extinct taxa that possessed intermediate breeding systems. Or perhaps the intermediate stages were transitory within ancestral species if the rate of character change exceeded the rate of speciation (cf. Frumhoff and Reeve 1994). These concerns are important, and the likelihood of having missed transitions needs to be evaluated on a case by case basis. In each case where we inferred a change from hermaphroditism to dioecy, the most obvious question concerns the likelihood that monoecy was an intermediate. We doubt that this is generally the case for several reasons. Monoecy is widely distributed in angiosperms and is characteristic of clades of many sizes, and we know of no reason to suppose that it is unstable or transitory from the standpoint of genetic and ecological mechanisms. Furthermore, models of change from monoecy to dioecy have generally envisioned a gradual process, involving alternating incorporation of genes that slightly affect male and female fertility (e.g., Charlesworth and Charlesworth 1978).

If monoecy is not likely as a transitory intermediate condition, what about other breeding systems? Although we do not observe changes to dioecy from gynodioecy, we believe that such transitions are a strong possibility based on several arguments. The direct origin of dioecy from hermaphroditism seems unlikely because this would require at least two mutations at once, one suppressing the development of the gynoecium and another suppressing the androecium. In theory, androdioecy (loss of gynoecia in some plants) could be an intermediary, but this condition is extremely rare in angiosperms, and it is unclear whether it has ever given rise to dioecy (e.g., Swensen et al. 1998). In contrast, gynodioecy is more common, and changes from hermaphroditism to dioecy through gynodioecy are well documented (e.g., Hart 1985; Weller et al.
1998). Most important for this argument, selection models based on nuclear and nuclear-cytoplasmic male sterility (Schultz 1994) suggest that gynodioecy may be unstable and may result in accelerated transitions to dioecy. A rapid transition from gynodioecy to dioecy within an ancestral species would not be detected in phylogenetic analysis.

Recent molecular studies also suggest that a hermaphroditic pathway may be important (e.g., DeLong et al. 1993). The simplest genetic models for induction of monoecy require at least two genes, which precisely affect the abortion of gynoecia and androecia in separate flowers on the same plant. Two genes are also required for induction of dioecy except that organ abortion is segregated among plants in this case. Monoecy is evidently not a prerequisite for the fixation of staminate or carpellate flowers within plants, and the view that monoecy is one step closer to dioecy than hermaphroditism does not draw support from current knowledge of genetic mechanisms or phylogenetic relationships.

\section{Conclusions}

We draw two main conclusions from this article. First, monocots illustrate how phylogenetic analysis of character change can lead to a conclusion that differs from interpretations based on taxonomic evidence (e.g., Lewis 1942) or rank-based correlations (Renner and Ricklefs 1995). If the fundamental question concerns sequences of character change, the most direct approach is a phylogenetic one. A taxonomic correlation may result for reasons other than a particular repeated sequence of character change. In this case we believe that the correlation between monoecy and dioecy is strong not because monoecy tends to give rise to dioecy but instead because changes in breeding system (in whatever direction) tend to be clustered in the tree. Our second conclusion concerns the evolution of plant breeding systems in general. Taking the main result of this analysis at face value, we suggest renewed attention to models and mechanisms of transition from hermaphroditism to dioecy, possibly through a transient gynodioecious phase.

\section{Acknowledgments}

We thank D. Ackerly, D. Baum, D. Ferguson, E. Kellogg, R. Ree, R. Spangler, and the students of Biology 216 (1995) for comments and helpful discussion. S. Renner and R. Ricklefs kindly made available the database of dioecious flowering plants used in their analyses, and A. Sakai and S. Weller sent us their manuscript on phylogenies and plant breeding systems. M. Pagel provided versions of his program Discrete for three- and four-state characters, and R. Ree assisted with the maximum likelihood analyses. 


\section{Literature Cited}

Ackerly, D. D., and M. J. Donoghue. 1998. Leaf size, sapling allometry, and Corner's rules: phylogeny and correlated evolution in maples (Acer). American Naturalist 152:767-791.

Baker, H. G. 1984. Some functions of dioecy in seed plants. American Naturalist 124:149-158.

Bawa, K. S. 1980. Evolution of dioecy in flowering plants. Annual Review of Ecology and Systematics 11:15-39.

- 1982. Outcrossing and the incidence of dioecism in island floras. American Naturalist 119:866-871.

Bierzychudek, P. 1984. Assessing "optimal” life histories in a fluctuating environment: the evolution of sexchanging by jack-in-the-pulpit. American Naturalist 123:829-840.

Bogler, D. J., and B. B. Simpson. 1996. Phylogeny of Agavaceae based on ITS rDNA sequence variation. American Journal of Botany 83:1225-1235.

Charlesworth, B., and D. Charlesworth. 1978. A model for the evolution of dioecy and gynodioecy. American Naturalist 112:975-997.

Charnov, E. L. 1982. The theory of sex allocation. Princeton University Press, Princeton, N.J.

Chase, M. W., M. R. Duvall, H. G. Hills, J. G. Conran, A. V. Cox, L. E. Eguiarte, J. Hartwell, et al. 1995a. Molecular phylogenetics of Lilianae. Pages 109-137 in P. J. Rudall, P. J. Cribb, D. F. Cutler, and C. J. Humphries, eds. Monocotyledons: systematics and evolution. Royal Botanic Gardens, Kew.

Chase, M. W., D. W. Stevenson, P. Wilkin, and P. J. Rudall. 1995b. Monocot systematics: a combined analysis. Pages 685-730 in P. J. Rudall, P. J. Cribb, D. F. Cutler, and C. J. Humphries, eds. Monocotyledons: systematics and evolution. Royal Botanic Gardens, Kew.

Collins, T. M., P. H. Wimberger, and G. P. Nayler. 1994. Compositional bias, character-state bias and character state reconstruction using parsimony. Systematic Biology 43:482-496.

Cox, P. A., K. L. Huynh, and B. C. Stone. 1995. Evolution and systematics of the Pandanaceae. Pages 663-684 in P. J. Rudall, P. B. Cribb, D. F. Cutler, and C. J. Humphries, eds. Monocotyledons: systematics and evolution. Royal Botanic Gardens, Kew.

Darwin, C. R. 1877. The different forms of flowers on plants of the same species. J. Murray, London.

DeLong, A., A. Calderon-Urrea, and S. L. Dellaporta. 1993. Sex determination gene TASSELSEED2 of maize encodes a short-chain alcohol dehydrogenase required for stage-specific floral organ abortion. Cell 74:757-768.

de Queiroz, A., M. J. Donoghue, and J. Kim. 1995. Separate versus combined analysis of phylogenetic evidence. Annual Review of Ecology and Systematics 26:657-681.
Donoghue, M. J. 1989. Phylogenies and the analysis of evolutionary sequences, with examples from seed plants. Evolution 43:1137-1156.

- 1994. Progress and prospects in reconstructing plant phylogeny. Annals of the Missouri Botanical Garden 81:405-418.

Donoghue, M. J., and D. A. Ackerly. 1996. Phylogenetic uncertainties and sensitivity analyses in comparative biology. Philosophical Transactions of the Royal Society of London Series B, Biological Sciences 351:1241-1249.

Donoghue, M. J., R. H. Ree, and D. A. Baum. 1998. Phylogeny and the evolution of flower symmetry in the Asteridae. Trends in Plant Science 3:311-317.

French, J. C., M. G. Chung, and Y. K. Hur. 1995. Chloroplast DNA phylogeny of the Ariflorae. Pages 255-275 in P. J. Rudall, P. B. Cribb, D. F. Cutler, and C. J. Humphries, eds. Monocotyledons: systematics and evolution. Royal Botanic Gardens, Kew.

Frumhoff, P. C., and H. K. Reeve. 1994. Using phylogenies to test hypotheses of adaptation: a critique of some current proposals. Evolution 48:172-180.

Givnish, T. J. 1980. Ecological constraints on the evolution of breeding systems in seed plants: dioecy and dispersal in gymnosperms. Evolution 34:959-972.

- 1982. Outcrossing versus ecological constraints in the evolution of dioecy. American Naturalist 119: 849-865.

Hart, J. A. 1985. Evolution of dioecism in Lepechinia Willd. sect. Parviflorae (Lamiaceae). Systematic Botany 10: 147-154.

Janz, N., and S. Nylin. 1998. Butterflies and plants: a phylogenetic study. Evolution 52:486-502.

Kellogg, E. A., and C. S. Campbell. 1987. Phylogenetic analyses of the Gramineae. Pages 310-322 in T. R. Soderstrom, K. W. Hilu, C. S. Campbell, and M. E. Barkworth, eds. Grass systematics and evolution. Smithsonian Institution, Washington, D.C.

Kellogg, E. A., and H. P. Linder. 1995. Phylogeny of Poales. Pages 511-542 in P. J. Rudall, P. J. Cribb, D. F. Cutler, and C. J. Humphries, eds. Monocotyledons: systematics and evolution. Royal Botanic Gardens, Kew.

Kellogg, E. A., and L. Watson. 1993. Phylogenetic studies of a large data set. I. Bambusoideae, Andropogonidae, and Pooidae (Graminae). Botanical Review 59:273-343.

Kohn, J. R., S. W. Graham, B. Morton, J. J. Doyle, and S. C. H. Barrett. 1996. Reconstruction of the evolution of reproductive characters in Pontederiaceae using phylogenetic evidence from chloroplast DNA restrictionsite variation. Evolution 50:1454-1469.

Kress, W. J. 1995. Phylogeny of the Zingiberanae: morphology and molecules. Pages 443-460 in P. J. Rudall, P. J. Cribb, D. F. Cutler, and C. J. Humphries, eds. 
Monocotyledons: systematics and evolution. Royal Botanic Gardens, Kew.

Le Roux, L. G., and E. A. Kellogg. 1999. Floral development and the formation of unisexual spikelets in the Andropogoneae (Poaceae). American Journal of Botany 86: 354-366.

Les, D. H., M. A. Cleland, and M. Waycott. 1997. Phylogenetic studies in Alismatidae. II. Evolution of marine angiosperms (seagrasses) and hydrophily. Systematic Botany 22:443-463.

Lewis, D. 1942. The evolution of sex in flowering plants. Biological Review of the Cambridge Philosophical Society $17: 46-67$.

Linder, H. P., and E. A. Kellogg. 1995. Phylogenetic patterns in the commelinid clade. Pages 473-496 in P. J. Rudall, P. J. Cribb, D. F. Cutler, and C. J. Humphries, eds. Monocotyledons: systematics and evolution. Royal Botanic Gardens, Kew.

Lloyd, D., G. 1982. Selection of combined versus separate sexes in seed plants. American Naturalist 120:571-585.

Losos, J. B. 1995. An approach to the analysis of comparative data when a phylogeny is unavailable or incomplete. Systematic Biology 43:117-123.

Maddison, D. R. 1994. Phylogenetic methods for inferring the evolutionary history and process of change in discretely valued characters. Annual Review of Entomology 39:267-292.

Maddison, W. 1989. Reconstructing character evolution on polytomous cladograms. Cladistics 5:365-377.

Maddison, W., and D. R. Maddison. 1992. MacClade: analysis of phylogeny and character evolution, Sinauer, Sunderland, Mass.

Mason-Gamer, R. J., and E. A. Kellogg. 1996. Testing for phylogenetic conflict among molecular data sets in the tribe Triticeae (Gramineae). Systematic Biology 45: 524-545.

Nixon, K. C., and J. I. Davis. 1991. Polymorphic taxa, missing values and cladistic analysis. Cladistics 7:233-241.

Pagel, M. 1994. Detecting correlated evolution on phylogenies: a general method for the comparative analysis of discrete characters. Proceedings of the Royal Society of London B, Biological Sciences 255:37-45.

- 1997. Inferring evolutionary processes from phylogenies. Zoologica Scripta 26:331-348.

Ree, R. H. and M. J. Donoghue. 1998. Step matrices and the interpretation of homoplasy. Systematic Biology 47: 582-588.

- 1999. Inferring rates of change in flower symmetry in asterid angiosperms. Systematic Biology 48: 633-641.

Renner, S. S., and R. E. Ricklefs. 1995. Dioecy and its correlates in the flowering plants. American Journal of Botany 82:596-606.
Rolland, C., E. Danchin, and M. de Fraipont. 1998. The evolution of coloniality in birds in relation to food, habitat, predation and life-history traits: a comparative analysis. American Naturalist 151:514-529.

Ross, M. D. 1982. Five evolutionary pathways to subdioecy. American Naturalist 119:297-318.

Rudall, P. J., P. J. Cribb, D. F. Cutler, and C. J. Humphries, eds. 1995. Monocotyledons: systematics and evolution, Royal Botanic Gardens, Kew.

Sakai, A. K., W. L. Wagner, D. M. Ferguson, and D. R. Herbst. 1995. Biogeographical and ecological correlates of dioecy in the Hawaiian flora. Ecology 76:2530-2543.

Sanderson, M. 1991. In search of homoplastic tendencies: statistical inference of topological patterns in homoplasy. Evolution 45:351-358.

Sanderson, M., A. Purvis, and C. Henze. 1998. Phylogenetic supertrees: assembling the trees of life. Trends in Ecology \& Evolution 13:105-109.

Schultz, S. T. 1994. Nucleo-cytoplasmic male sterility and alternative routes to dioecy. Evolution 48:1933-1945.

Schultz, T. R., R. B. Cocroft, and G. A. Churchill. 1996. The reconstruction of ancestral character states. Evolution 50:504-511.

Sillen-Tullberg, B. 1988. Evolution of gregariousness in aposematic butterfly larvae: a phylogenetic analysis. Evolution 42:293-305.

-1993. The effect of biased inclusion of taxa on the correlation between discrete characters in phylogenetic trees. Evolution 47:1182-1191.

Simpson, D. 1995. Relationships within Cyperales. Pages 497-509 in P. J. Rudall, P. J. Cribb, D. F. Cutler, and C. J. Humphries, eds. Monocotyledons: systematics and evolution. Royal Botanic Gardens, Kew.

Simpson, M. G. 1990. Phylogeny and classification of the Haemodoraceae. Annals of the Missouri Botanical Garden 77:722-784.

Stevenson, D. W., and H. Loconte. 1995. Cladistic analysis of monocot families. Pages 543-578 in P. J. Rudall, P. B. Cribb, D. F. Cutler, and C. J. Humphries, eds. Monocotyledons: systematics and evolution. Royal Botanic Gardens, Kew.

Swensen, S. M., J. N. Luthi, and L. H. Rieseberg. 1998. Datiscaceae revisited: monophyly and the sequence of breeding system evolution. Systematic Botany 23: 157-169.

Swofford, D. L., and W. P. Maddison. 1992. Parsimony, character-state reconstructions, and evolutionary inferences. Pages 186-223 in R. L. Mayden, ed. Systematics, historical ecology, and North American freshwater fishes. Stanford University Press, Stanford, Calif.

Thomson, J. D., and S. C. H. Barrett. 1981. Selection for outcrossing, sexual selection, and the evolution of dioecy in plants. American Naturalist 118:443-449. 
Thomson, J. D., and J. Brunet. 1990. Hypotheses for the evolution of dioecy in seed plants. Trends in Ecology \& Evolution 5:11-16.

Uhl, N. W., J. Dransfield, J. I. Davis, M. A. Luckow, K. S. Hansen, and J. J. Doyle. 1995. Phylogenetic relationships among palms: cladistic analyses of morphological and chloroplast DNA restriction site variation. Pages 623-661 in P. J. Rudall, P. B. Cribb, D. F. Cutler and C. J., Humphries, eds. Monocotyledons: systematics and evolution. Royal Botanic Gardens, Kew.

Van den Borre, A. 1994. Taxonomy of the Chloridoideae (Poaceae), with special reference to the genus Eragrostis. Ph.D. thesis, Australian National University.

Watson, L., and M. J. Dallawitz. 1992. The grass genera of the world. C.A.B. International, Wallingford.

Weller, S. G., and A. Sakai. 1999. Using phylogenetic approaches for the analysis of plant breeding system evolution. Annual Review of Ecology and Systematics (in press).

Weller, S. G., M. J. Donoghue, and D. Charlesworth. 1995. The evolution of self-incompatibility in flowering plants: a phylogenetic approach. Pages 355-382 in P. C. Hoch and A. G. Stephenson, eds. Experimental and molecular approaches to plant biosystematics. Missouri Botanical Garden, St. Louis.

Weller, S. G., A. K. Sakai, A. E. Rankin, A. Golonka, B. Kutcher, and K. E. Ashby. 1998. Dioecy and the evolution of pollination systems in Schiedea and Alsinidendron (Caryophyllaceae: Alsinoideae) in the Hawaiian Islands. American Journal of Botany 85:1377-1388.

Westergaard, M. 1958. The mechanism of sex determination in dioecious flowering plants. Advances in Genetics 9:217-281.

Willson, M. F. 1982. Sexual selection and dicliny in angiosperms. American Naturalist 119:579-583.

- 1991. Sexual selection, sexual dimorphism, and plant phylogeny. Evolutionary Ecology 5:69-87.

Yampolsky, C., and H. Yampolsky. 1922. Distribution of sex forms in the phanerogamic flora. Bibliotheca Genetica 3:1-62. 